\title{
DESUSPENDABILITY OF FREE INVOLUTIONS \\ ON BRIESKORN SPHERES
}

\author{
BY CHARLES H. GIFFEN ${ }^{1}$
}

Communicated by William Browder, September 5, 1968

The Brieskorn manifolds $K_{a}^{2 m-1}$ which we consider are the following. Let $V_{q}(m)$ be the set of points $\left(z_{0}, \cdots, z_{m}\right) \in C^{m+1}$ such that

$$
z_{0}^{q}+\sum_{i=1}^{m} z_{i}^{2}=0 \text {, }
$$

and let $S^{2 m+1} \subset C^{m+1}$ be the unit sphere; then

$$
K_{q}^{2 m-1}=V_{q}(m) \cap S^{2 m+1}
$$

is a smooth (actually, weakly complex) codimension 2 submanifold of $S^{2 m+1}$ (c.f. [2]). If we set

$$
F_{q}^{2 m}=\left\{\left(z_{0}, \cdots, z_{m}\right) \in S^{2 m+1} \mid z_{0}^{q}+\sum_{i=1}^{m} z_{i}^{2} \geqq 0\right\},
$$

then $\left(S^{2 m+1}, K_{q}^{2 m-1}\right)$ is a fibered pair over $S^{1}$ in the sense of [4] with parallelizable fiber $F_{q}^{2 m}$; moreover, $K_{\mathscr{2} d+1}^{-4 k+1}$ is a homotopy sphere, and the Arf invariant $c\left(F_{2 d+1}^{4 k+2}\right) \in Z_{2}$ is (cf. [8]):

$$
\begin{aligned}
& 0 \quad \text { if } d \equiv 0,3 \bmod 4, \\
& 1 \quad \text { if } d \equiv 1,2 \bmod 4 .
\end{aligned}
$$

The involution $T: C^{m+1} \rightarrow C^{m+1}$ given by $T\left(z_{0}, z\right)=\left(z_{0},-z\right)$ is fixed point free on $K_{a}^{2 m-1}$. The weakly complex bordism classification of $\left(T, K_{q}^{2 m-1}\right)$ is considered in $[5]$. We shall have need here of the invariant codimension 1 submanifold $K_{q}^{2 m}$ of $K_{q}^{2 m+1}$ given by

$$
K_{q}^{2 m}=\left\{\left(z_{0}, \cdots, z_{m+1}\right) \in K_{q}^{2 m+1} \mid z_{m+1} \text { imaginary }\right\} .
$$

Regarding $C^{m+1} \subset C^{m+2}$ in the obvious way, we have

$$
\left(T, K_{q}^{2 m-1}\right) \subset\left(T, K_{q}^{2 m}\right) \subset\left(T, K_{q}^{2 m+1}\right) .
$$

Setting $Q_{a}^{n}=K_{a}^{n} / T$, we see that there is a homotopy equivalence of degree \pm 1

$$
h: Q_{2 d+1}^{4 k+1} \rightarrow P^{4 k+1}
$$

1 Research supported by N.S.F. grant GP-6567. 
transverse regular to $P^{n} \subset P^{4 k+1}$ for any $n \leqq 4 k$, such that $h^{-1}\left(P^{n}\right)$ $=Q_{2 n+1}^{n}$. So we have $h: Q_{2 d+1}^{n} \rightarrow P^{n}$ of degree \pm 1 for all $n$ (use twisted coefficients if $n$ is even), and the tangent bundle of $Q_{2 q+1}^{n}$ is induced from a bundle over $P^{n}$ (since this is true for $n=4 k+1$ when $h$ is a homotopy equivalence).

In [3] there is defined an Arf invariant $c\left(T, \Sigma^{4 k+1}\right) \in Z_{2}$ where $T$ is a free smooth or PL involution on the homotopy (or homology) $(4 k+1)$-sphere $\Sigma^{4 k+1}$ and $k>0$; if $\Sigma^{4 k+1}$ is a homotopy sphere and $k>1$, then $c\left(T, \Sigma^{4 k+1}\right)=0$ iff $\left(T, \Sigma^{4 k+1}\right)$ desuspends (i.e. has an invariant codimension 1 sphere). In particular, $c\left(T, K_{2 d+1}^{4 k+1}\right)$ is defined for $k>0$.

In $[9$, Theorems $4.14,6.8]$ there is defined an invariant $c\left(Q_{2 d+1}^{n}, h\right)$ $\in Z_{2}$ for $n>1, n \neq 1 \bmod 4$; if $n>5$, this invariant vanishes iff surgery can be done to make $h$ a homotopy equivalence.

The following is the principal result of this paper.

(1) Theorem. The invariants $c\left(F_{2 d+1}^{4 k+2}\right), c\left(T, K_{2 d+1}^{4 k+1}\right), c\left(Q_{2 d+1}^{n}, h\right)$ for $n \neq 1 \bmod 4$ are all equal and given by formula $(*)$.

(2) REMARKs. This provides many examples of nondesuspendable free smooth involutions on homotopy $(4 k+1)$-spheres, $k>0$. The example of Berstein [1] can be shown to be diffeomorphic to $\left(T, K_{3}^{4 k+1}\right)$.

That $c\left(F_{2 d+1}^{4 k+2}\right)=c\left(T, K_{2 d+1}^{4 k+1}\right)$ is only seen through a coincidence of computations arising in this example. In particular, we do not prove the conjecture that if $\Theta_{4 k+1}(\partial \Pi) \neq 0$, then $\left(T, \Sigma^{4 k+1}\right)$ desuspends iff $\Sigma^{4 k+1}$ is a standard sphere.

Computations that show $c\left(Q_{2 d+1}^{4 k-1}, h\right)$ is given by $(*)$ have been known to the author for a long time. The original one used techniques similar to [6] and was excessively complicated. A much shorter computation for $c\left(Q_{2 d+1}^{4 k-1}, h\right)$ can be made using [9, Summary 6.9]; for, if

$$
\Delta(t)=t^{2 d}+t^{2 d-1}+\cdots+1 \in Z\left[t, t^{-1}\right]
$$

denotes the characteristic polynomial of $K_{2 a+1}^{4 k+1} \subset S^{4 k+1}$ (c.f. [8]), then the image $\tilde{\Delta}(t) \in Z\left(Z_{2}\right)$ under the obvious ring epimorphism

$$
Z\left[t, t^{-1}\right] \rightarrow Z\left(Z_{2}\right)
$$

can be shown by an uncomplicated geometric argument to be exactly the determinant of $H_{2 k-1}\left(K_{2 d+1}^{4 k-1}\right)$ as a $Z\left(Z_{2}\right)$-module.

For the proof of the theorem, we give here the even simpler and more comprehensible calculation that $c\left(Q_{2 d+1}^{4 k-2}, h\right)$ is given by (*). Specifically, the map

$$
e: \quad\left(K_{q}^{2 m+1}, K_{q}^{2 m-1}\right) \rightarrow\left(S^{2 m+1}, K_{q}^{2 m-1}\right)
$$


given by $e\left(z, z_{m+1}\right)=z /|z|$ is the 2 -fold cyclic branched covering, and $e^{-1}\left(F_{a}^{2 m}\right)=K_{a}^{2 m}$. In fact,

$$
\Phi_{q}^{2 m}=\left\{\left(z, z_{m+1}\right) \in K_{q}^{2 m+1} \mid \operatorname{Im}\left(z_{m+1}\right) \geqq 0\right\}
$$

is a characteristic submanifold of $\left(T, K_{q}^{2 m}\right)$, for

$$
K_{q}^{2 m}=\Phi_{q}^{2 m} \cup T \Phi_{q}^{2 m}, \quad K_{q}^{2 m-1}=\Phi_{q}^{2 m} \cap T \Phi_{q}^{2 m} .
$$

Moreover, $e: \Phi_{q}^{2 m} \rightarrow F_{q}^{2 m}$ is a diffeomorphism, and $\Phi_{q}^{2 m}$ inherits a framing from that of $F_{q}^{2 m}$ so that $c\left(F_{2 d+1}^{4 k-2}\right)=c\left(\Phi_{2 d+1}^{4 k-2}\right)$. We see that $Q_{q}^{2 m}$ is obtained from $Q_{q}^{2 m-1}$ by attaching $\Phi_{q}^{2 m}$ (or $F_{q}^{2 m}$ ) to $Q_{q}^{2 m-1}$ by the double covering $K_{q}^{2 m-1} \rightarrow Q_{q}^{2 m-1}$. Now as $h: Q_{2 d+1}^{4 k-2} \rightarrow P^{4 k-3}$ restricts to the homotopy equivalence $h: Q_{2 d+1}^{4 k-3} \rightarrow P^{4 k-3}$, it follows from [9, Theorem 4.14] and the correspondence between surgery obstructions induced by $\{1\} \rightarrow Z_{2}$ (cf. $[9$, p. 272]) that

$$
c\left(Q_{2 d+1}^{4 k-2}, h\right)=c\left(F_{2 d+1}^{4 k-2}\right),
$$

which as remarked, is given by $(*)$.

To complete the proof of the theorem, we need the equalities

$$
c\left(T, K_{2 d+1}^{4 k+1}\right)=c\left(Q_{2 d+1}^{4 k}, h\right)=c\left(Q_{2 d+1}^{4 k-1}, h\right)=c\left(Q_{2 d+1}^{4 k-2}, h\right) .
$$

The first is proved in $[1, \S 1]$ and also asserted in [7, Theorem $5 f$. and [10, Remark 4]. The last two equalities are consequences of [10, Lemma 3] and are implicit in the results of [7]. This proves the theorem.

As corollary to the proof of (1), we have the following result. Let $\left(T, \Sigma^{4 k+1}\right)$ be a smooth free involution on the homotopy $(4 k+1)$ sphere $\Sigma^{4 k+1}$ and set $Q^{4 k+1}=\Sigma^{4 k+1} / T$. We suppose $k>0$.

(3) Proposition. There is a homotopy equivalence

$$
h: Q^{4 k+1} \rightarrow P^{4 k+1}
$$

transverse regular to $P^{4 k-3}$ and $P^{4 k-2}$ with $Q^{4 k-3}=h^{-1}\left(P^{4 k-3}\right)$ homotopy equivalent to $P^{4 k-3}$; for any such $h, Q^{4 k-2}=h^{-1}\left(P^{4 k-2}\right)$ is obtained from $Q^{4 k-3}$ by attaching to $Q^{4 k-3}$ a framed $(4 k-2)$-manifold $F^{4 k-2}$ with homotopy sphere boundary $\Sigma^{4 k-3}$ via a smooth double covering $\Sigma^{4 k-3}$ $\rightarrow Q^{4 k-3}$. The invariant $c\left(Q^{4 k-2}, h\right)=c\left(F^{4 k-2}\right)=c\left(T, \Sigma^{4 k+1}\right)$.

(4) CoRollary. With $\left(T, \Sigma^{4 k+1}\right)$ as above and $\Theta_{4 k-3}(\partial \Pi) \neq 0$, then the universal covering $\Sigma^{4 k-3}$ of any $Q^{4 k-3}$ obtained as in (3) is an exotic $\pi$-boundary iff $c\left(T, \Sigma^{4 k+1}\right)=1$; otherwise, $\Sigma^{4 k-3} \approx S^{4 k-3}$.

(5) REMARK. In [10, Remark 4] it is asserted that the invariants 
of [3] provide an obstruction theory for the kernel and cokernel of the suspension

$$
\Sigma: \quad I_{n} \rightarrow I_{n+1}
$$

of PL homotopy structures on projective spaces. This is clearly true for the cokernel. It also happens to be true for the kernel if $n \neq 1$ $\bmod 4$. However, if $n=4 k+1$ it is false that the uniqueness Arf invariant of [3] is an invariant of the kernel of $\Sigma$. In terms of homotopy projective spaces (smooth or PL) $Q_{0}^{4 k+1}, Q_{1}^{4 k+1} \subset Q^{4 k+2}$ this Arf invariant is nonzero iff in $Q^{4 k+2} \times I$ there is a cobordism $W^{4 k+2}$ from $Q_{0}^{4 k+1} \times 0$ to $Q_{1}^{4 k+1} \times 1$ of the form

$$
Q_{0}^{4 k+1} \times I \#{ }^{4 k+2},
$$

where $F^{4 k+2}$ is a parallelizable $(4 k+2)$-manifold with homotopy sphere boundary, $c\left(F^{4 k+2}\right)=1$, and where \# denotes boundary connected sum. Hence, in the smooth case $Q_{0}^{4 k+1} \approx Q_{1}^{4 k+1} \# \Sigma^{4 k+1}$ where $\Sigma^{4 k+1}$ is a generator of $\Theta_{4 k+1}(\partial \Pi)$; combinatorially, $Q_{0}^{4 k+1}$ and $Q_{1}^{4 k+1}$ are the same. The author understands that Berstein and Livesay have constructed just such an example.

(6) Additional Comments. W. Browder remarks that he has an independent and quite different proof of (1), using his general setting for the Arf invariant. He also says that S. Lopez de Medrano's thesis results contain (3) and (4) as well as the Berstein-Livesay example.

\section{REFERENCES}

1. I. Berstein, Involutions with nonzero Arf invariant, Bull. Amer. Math. Soc. 74 (1968), 678-682.

2. E. V. Brieskorn, Examples of singular normal complex spaces which are topological manifolds, Proc. Nat. Acad. Sci. U.S.A. 55 (1966), 1395-1397.

3. W. Browder and G. R. Livesay, Fixed point free involutions on homotopy spheres, Bull. Amer. Math. Soc. 73 (1967), 242-245. 198.

4. C. H. Giffen, The generalized Smith conjecture, Amer. J. Math. 88 (1966), 187-

5. - Weakly complex involutions and cobordism of projective spaces (to appear).

6. J. Levine, Polynomial invariants of knots of codimension two, Ann. of Math. (2) 84 (1966), 537-554.

7. S. Lopez de Medrano, Some results on involutions of homotopy spheres, Proceedings of the Tulane conference on transformation groups, 1967 (to appear).

8. J. W. Milnor, On isolated singularities of hypersurfaces (mimeographed).

9. C. T. C. Wall, Surgery of non-simply connected manifolds, Ann. of Math. 84 (1966), 217-276.

10. - Free piecewise linear involutions on spheres, Bull. Amer. Math. Soc. 74 (1968), 554-558.

University of Virginia, Charlottesville, Virginia 22901 